\title{
An example of a convex body without symmetric projections.
}

\author{
E. D. Gluskin A. E. Litvak* N. Tomczak-Jaegermann
}

\begin{abstract}
Many crucial results of the asymptotic theory of symmetric convex bodies were extended to the non-symmetric case in recent years. That led to the conjecture that for every $n$-dimensional convex body $K$ there exists a projection $P$ of rank $k$, proportional to $n$, such that $P K$ is almost symmetric. We prove that the conjecture does not hold. More precisely, we construct an $n$-dimensional convex body $K$ such that for every $k>C \sqrt{n \ln n}$ and every projection $P$ of rank $k$, the body $P K$ is very far from being symmetric. In particular, our example shows that one can not expect a formal argument extending the "symmetric" theory to the general case.
\end{abstract}

\section{Introduction}

The asymptotic theory of finite dimensional normed spaces studies the behavior of various functionals on the set of all finite dimensional normed spaces, or equivalently, the behavior of centrally-symmetric convex bodies in $\mathbb{R}^{n}$, when the dimension grows to infinity. The development of the theory in the last years required to abandon the condition of central symmetry. It turns out that many results of the theory remain valid in the non-symmetric case, although their proofs require new and different arguments (see e.g., [GGM], [LMP], [MP], [R1], [R2], [LT] and references therein). Unexpected similarity of results concerning general convex bodies and their counterparts in the centrally symmetric case lead a number of authors to the following conjecture which we state a bit vaguely.

\footnotetext{
*This author holds Lady Davis Fellowship.
} 
Conjecture. For every convex body $K$ in $\mathbb{R}^{n}$ there exists an orthogonal projection $P$ of rank $k$, proportional to $n$, such that $P K$ is almost symmetric.

If this conjecture were true, an extension of large class of problems in the asymptotic theory of finite dimensional normed spaces to the non-symmetric case would be automatic. However, it is not so, and in this note we construct a counterexample. Our ideas and intuitions have two sources. The first are constructions of random symmetric convex bodies that have been initiated in [G1] (an exhaustive survey of this direction can be found in [MT]). In particular, the main line of the present argument is close to [G2]. The second source is a recent paper [LT] where it was shown, among other results, that a random projection of a simplex is very far from being symmetric. More precisely, if $P$ is a random orthogonal projection of rank $k>\ln n$ and $S \subset \mathbb{R}^{n}$ is the regular simplex then the distance from $P S$ to any centrally symmetric body is, with high probability, larger than $c \sqrt{k / \ln n}$, where $c$ is an absolute constant. This shows that the conjecture fails for random projections. It should be noted, however, that there exist projections of the simplex of proportional dimension which are close to symmetric ([GGM]). We will show in the present note that there exists a convex body $K \subset \mathbb{R}^{n}$ such that its image under any rank $k$ projection, with $k>\sqrt{n \ln n}$, has the distance from any centrally symmetric body larger than $c k / \sqrt{n \ln n}$.

\section{Definitions, notations, known results.}

We will use the standard notation from the local theory of Banach spaces (see e.g. $[\mathrm{MS}]$ ). Given a finite set $\mathcal{N}$, its cardinality is denoted by $|\mathcal{N}|$. Denote the canonical Euclidean norm on $\mathbb{R}^{n}$ by $|\cdot|$, the Euclidean unit ball by $B_{2}^{n}$, and the Euclidean unit sphere by $S^{n-1}$. The distance between a point $a \in \mathbb{R}^{n}$ and a set $K \subset \mathbb{R}^{n}$ is

$$
\operatorname{dist}(a, K)=\min \{|a-x| \mid x \in K\} .
$$

By a convex body $K \subset \mathbb{R}^{n}$ we shall always mean a compact convex set with the non-empty interior, and without loss of generality we shall assume that $0 \in \operatorname{Int} K$. The gauge of $K$ is denoted by $\|\cdot\|_{K}$, i.e., $\|x\|_{K}=$ $\inf \{\lambda>0 \mid x \in \lambda K\}$.

Given convex bodies $K, L$ in $\mathbb{R}^{n}$, we define the geometric distance by

$$
\widetilde{d}(K, L)=\inf \{\alpha \beta \mid \alpha>0, \beta>0,(1 / \beta) L \subset K \subset \alpha L\} .
$$


The Banach-Mazur distance is defined by

$$
d(K, L)=\inf \{\widetilde{d}(u(K-z), L-x)\},
$$

where infimum is taken over all $z, x \in \mathbb{R}^{n}$ and all linear operators $u: \mathbb{R}^{n} \rightarrow$ $\mathbb{R}^{n}$. Clearly, if $K$ and $L$ are centrally symmetric, then the infimum is attained at $z=x=0$. Thus, in the centrally symmetric case the definition coincides with the standard definition of the Banach-Mazur distance between balls of normed spaces. Moreover, if $L$ is centrally symmetric then

$$
d(K, L) \leq 2 \inf \{\widetilde{d}(u(K-z), L)\} .
$$

For simplicity we will use $d(K, L)$ for $\inf \{\widetilde{d}(u(K-z), L)\}$ in the case $L=$ $-L$.

Denote by $\mathcal{C}^{n}$ the set of all centrally symmetric bodies in $\mathbb{R}^{n}$. Let $K$ be a convex body in $\mathbb{R}^{n}$. The quantity

$$
d\left(K, \mathcal{C}^{n}\right)=\inf \left\{d(K, B) \mid B \in \mathcal{C}^{n}\right\}
$$

measures, in a sense, the "non-symmetry" of $K$. (See [Gr], where the problem of the measure of "non-symmetry" is discussed.) By compactness, there exist $a \in K$ and a centrally symmetric convex body $B \subset \mathbb{R}^{n}$ such that $d\left(K, \mathcal{C}^{n}\right)=\widetilde{d}(K-a, B)$. Observe that we also have

$$
\begin{aligned}
d\left(K, \mathcal{C}^{n}\right) & =\widetilde{d}(K-a,(K-a) \cap-(K-a)) \\
& =\widetilde{d}(K-a, \operatorname{conv}\{(K-a) \cup-(K-a)\}) .
\end{aligned}
$$

That is, $(K-a) \cap-(K-a)$ and conv $\{(K-a) \cup-(K-a)\}$ are two centrally symmetric bodies closest to $K$. Also note that for any $a \in K$ we have

$$
\frac{1}{2}(K-K) \subset \operatorname{conv}\{(K-a) \cup-(K-a)\} \subset K-K .
$$

Given a convex body $K \subset \mathbb{R}^{n}$ and $A \geq 1$, we say that $K$ is $A$-symmetric if $d\left(K, \mathcal{C}^{n}\right) \leq A$, and that $K$ is $A$-symmetric with respect to a center $a \in \mathbb{R}^{n}$ if $\widetilde{d}(K-a, B) \leq A$ for some centrally symmetric body $B$. We also denote $K-a$ by $K_{a}$. By the above remark, the body $K$ is $A$-symmetric with respect to a center $a \in \mathbb{R}^{n}$ if and only if $\|-x\|_{K_{a}} \leq A\|x\|_{K_{a}}$ for every $x \in \mathbb{R}^{n}$.

For a linear operator $u: \mathbb{R}^{n} \rightarrow \mathbb{R}^{n}$, by $\|u\|$ we denote its operator norm $\left\|u: \ell_{2}^{n} \rightarrow \ell_{2}^{n}\right\|$. Finally, the letters $C, c, c_{0}, c_{1}, \ldots$ denote absolute constants whose values may be different from line to line. 
We shall now describe our probabilistic setting and the background results. Let us note at the beginning that we shall work with random vectors uniformly distributed on the sphere; however, similar calculations could be done with random Gaussian vectors, similarly as e.g., in [MT].

As usual, $\mathbf{E}$ denotes the expectation and $\mathbf{P r}$ denotes the probability. Let $\omega$ be a random vector uniformly distributed on $S^{n-1}$ and $P$ be an orthogonal projection of rank $1 \leq k<n$. The following well-known inequalities easily follow from the concentration of measure phenomena (see e.g., [MS]). There is an absolute constant $c$ such that

$$
\operatorname{Pr}\left(\left\{\frac{\sqrt{k}}{2 \sqrt{n}} \leq|P \omega| \leq \frac{2 \sqrt{k}}{\sqrt{n}}\right\}\right) \geq 1-e^{-c k},
$$

and for every $t>0$,

$$
\operatorname{Pr}(\{|P \omega| \geq(1+t) \sqrt{k / n}\}) \leq e^{-c k t^{2}} .
$$

The direct computations show that for every $p>1$ and for every $x \in B_{2}^{n}$

$$
\left(\mathbf{E}|\langle\omega, x\rangle|^{p}\right)^{1 / p} \leq c \sqrt{p / n},
$$

where $c$ is an absolute constant. It follows then that for every integer $m$ and every $\left\{x_{i}\right\}_{i \leq m} \subset B_{2}^{n}$ we have

$$
\begin{aligned}
\mathbf{E} \max _{i \leq m}\left|\left\langle\omega, x_{i}\right\rangle\right| & \leq \mathbf{E}\left(\sum_{i=1}^{m}\left|\left\langle\omega, x_{i}\right\rangle\right|^{p}\right)^{1 / p} \\
& \leq\left(\mathbf{E} \sum_{i=1}^{m}\left|\left\langle\omega, x_{i}\right\rangle\right|^{p}\right)^{1 / p} \leq c m^{1 / p} \sqrt{p / n}
\end{aligned}
$$

Choosing $p=\ln m$ we obtain that there is an absolute constant $c_{0}$ such that

$$
\mathbf{E} \max _{i \leq m}\left|\left\langle\omega, x_{i}\right\rangle\right| \leq c_{0} \sqrt{\frac{\ln m}{n}} .
$$

for every every $\left\{x_{i}\right\}_{i \leq m} \subset B_{2}^{n}$. Of course the same estimate holds for any random vector $\omega$ taking values in $B_{2}^{n}$, whose distribution is invariant under rotations.

Let $(\Omega, \mathcal{F}$, Pr $)$ be a probability space. Let $\emptyset \subset \mathcal{F}_{1} \subset \mathcal{F}_{2} \subset \ldots \subset \mathcal{F}$ be $\sigma$-fields. Let $f_{0}, f_{1}, f_{2}, \ldots$ be a sequence of random variables such that $f_{i}$ is 
measurable with respect to $\mathcal{F}_{i}$ for every $i$, and the conditional expectation $\mathbf{E}\left(f_{i} \mid \mathcal{F}_{i-1}\right)=f_{i-1}$. Then the sequence $\left\{f_{i}\right\}$ is called a martingale with respect to $\left\{\mathcal{F}_{i}\right\}$.

We shall use the following well-known estimate for large deviation of martingales with bounded martingale differences. (As usual $\|d\|_{\infty}$ denotes the essential supremum of the absolute value of a random variable $d$ ).

Lemma $1.1([\mathrm{~A}])$ Let $0=f_{0}, f_{1}, \ldots, f_{m}$ be a martingale and let $d_{i}=$ $f_{i}-f_{i-1}$ be bounded for every $i \leq m$. Then for every positive $\lambda$ one has

$$
\operatorname{Pr}\left(\left\{\sum_{i=1}^{m} d_{i} \geq \lambda\right\}\right) \leq e^{-\lambda^{2} /(2 M)}
$$

where $M=\sum_{i=1}^{m}\left\|d_{i}\right\|_{\infty}^{2}$.

Let $h_{1}, \ldots, h_{m}$ be independent identically distributed random variables with $\mathbf{E} h_{i}=a$ (where $a>0$ ) and $\left\|h_{i}\right\|_{\infty} \leq b$. Applying Lemma 1.1 to $f_{i}=\sum_{j=1}^{i}\left(a-h_{i}\right)$ and $\lambda=m a / 2$ we get

$$
\operatorname{Pr}\left(\left\{\sum_{i=1}^{m} h_{i} \leq m a / 2\right\}\right) \leq e^{-m a^{2} /\left(32 b^{2}\right)}
$$

Finally we need the estimates for cardinality of nets in Banach spaces. Recall that a set $\mathcal{N}$ is called an $\varepsilon$-net for the set $K$ with respect to given norm $\|\cdot\|$ if for every $x \in K$ there is $a \in \mathcal{N}$ such that $\|x-a\| \leq \varepsilon$. The following fact gives a standard estimate for cardinality of nets (see e.g., [MT], Lemma 6).

Fact 1.2 Let $\|\cdot\|$ be a norm on $\mathbb{R}^{n}$ and $B$ be its unit ball. Then for every $0<\varepsilon \leq 1$ there is $\mathcal{N}$, an $\varepsilon$-net for the $B$ with respect to the norm $\|\cdot\|$, such that $|\mathcal{N}| \leq(3 / \varepsilon)^{n}$.

Remark. It is well known and easy to check that if $\mathcal{N}$ is an $\varepsilon$-net in $B$ in the norm $\|\cdot\|$, then for any subset $K \subset B$ there exists an $(2 \varepsilon)$-net $\mathcal{N}_{0} \subset K$ with cardinality $|\mathcal{N}|=\left|\mathcal{N}_{0}\right|$. Consider the case when $B$ is the set of all linear operators $u: \mathbb{R}^{n} \rightarrow \mathbb{R}^{n}$ satisfying $\left\|u: \ell_{2}^{n} \rightarrow \ell_{2}^{n}\right\| \leq 1$, and $K$ is the set of all orthogonal projections on $\mathbb{R}^{n}$ of rank $k$ (for a fixed $1 \leq k \leq n$ ). Thus there exists an $\varepsilon$-net $\mathcal{N}$ consisting of orthogonal projections of rank $k$ and such that $|\mathcal{N}| \leq(6 / \varepsilon)^{n^{2}}$. This is a very rough estimate, however it is sufficient for our purposes in this paper. We refer the interested reader to $[\mathrm{Sz}]$ where the investigation of $\varepsilon$-nets for the set of all rank $k$ orthogonal projections on $\mathbb{R}^{n}$ was done in detail. 


\section{An example}

Theorem 2.1 Let $n$ be a positive integer. There exist a convex body $K \subset \mathbb{R}^{n}$ such that for every projection $P$ the distance of $P K$ from the set of centrally symmetric convex bodies is bounded from below by

$$
d\left(P K, \mathcal{C}^{k}\right) \geq c \frac{k}{\sqrt{n \ln n}},
$$

where $k=\operatorname{rank} P$ and $c>0$ is an absolute constant.

Remark. Since clearly $d\left(P K, \mathcal{C}^{k}\right) \geq 1$, the theorem is of interest for $k \geq$ $\sqrt{n \ln n} / c$ only.

Lemma 2.2 Let $k \leq m$ be positive integers. Let $v_{1}, \ldots, v_{m}$ be independent identically distributed random vectors in $\mathbb{R}^{k}$ with rotation invariant distribution. Let $w_{1}, \ldots, w_{m}$ be random vectors in $\mathbb{R}^{k}$ such that $v_{i}$ is independent of $\left\{w_{1}, \ldots, w_{i-1}\right\}$ for every $i \leq m$. Assume that $v_{i}$ 's are distributed in $R B_{2}^{k}$ and $w_{i}$ 's are distributed in $r B_{2}^{k}$ for some $R, r>0$. Then

$$
\operatorname{Pr}\left(\left\{\sum_{i=1}^{m} \max _{j<i}\left|\left\langle v_{i}, w_{j}\right\rangle\right| \geq \operatorname{Rr}\left(\lambda+c_{0} m \sqrt{(\ln m) / k}\right)\right\}\right) \leq e^{-\lambda^{2} /(8 m)},
$$

where $c_{0}$ is the constant from (3).

Proof: Denote $f_{i}=\max _{j<i}\left|\left\langle v_{i}, w_{j}\right\rangle\right|$. By conditions of the lemma we have $\left|\left\langle v_{i}, w_{j}\right\rangle\right| \leq R r$. Let $\mathcal{F}_{i}$ be the $\sigma$-algebra generated by $v_{1}, \ldots, v_{i}, w_{1}, \ldots, w_{i}$. Let $\bar{f}_{i}$ be the conditional expectation $\mathbf{E}\left(f_{i} \mid \mathcal{F}_{i-1}\right)$ and $d_{i}=f_{i}-\bar{f}_{i}$. Then $u_{i}=$ $\sum_{j=1}^{i} d_{j}, i \leq m$, is a martingale with respect to $\left\{\mathcal{F}_{i}\right\}$. Since $\left\|d_{i}\right\|_{\infty} \leq 2 R r$, $i \leq m$, by Lemma 1.1 we obtain that

$$
\operatorname{Pr}\left(\left\{u_{m} \geq \operatorname{Rr} \lambda\right\}\right) \leq e^{-\lambda^{2} /(8 m)} .
$$

By (3), we have $\bar{f}_{i} \leq c_{0} R r \sqrt{(\ln m) / k}$, for $i \leq m$. Thus

$$
\begin{aligned}
& \operatorname{Pr}\left(\left\{\sum_{i=1}^{m} \max _{j<i}\left|\left\langle v_{i}, w_{j}\right\rangle\right| \geq \operatorname{Rr}\left(c_{0} m \sqrt{(\ln m) / k}+\lambda\right)\right\}\right) \\
& =\operatorname{Pr}\left(\left\{u_{m}+\sum_{i=1}^{m} \bar{f}_{i} \geq \operatorname{Rr}\left(c_{0} m \sqrt{(\ln m) / k}+\lambda\right)\right\}\right) \\
& \leq \operatorname{Pr}\left(\left\{u_{m} \geq \operatorname{Rr} \lambda\right\}\right)+\operatorname{Pr}\left(\left\{\sum_{i=1}^{m} \bar{f}_{i}>\operatorname{Rr} c_{0} m \sqrt{(\ln m) / k}\right\}\right) \\
& \leq e^{-\lambda^{2} /(8 m)} .
\end{aligned}
$$


This proves the lemma.

Remark. In the sequel we shall use the lemma as follows. Let $h_{1}, \ldots, h_{m}$ be independent identically distributed random vectors in $\mathbb{R}^{k}$ with rotation invariant distribution. Let $x \in \mathbb{R}^{k}$. Assume that $h_{i}$ 's are distributed in $r B_{2}^{k}$. Applying the lemma for $w_{i}=h_{i}-x$ and $v_{i}=\chi_{R B_{2}^{k}} \cdot h_{i}$, where $\chi_{K}$ denotes the indicator of the set $K$, we obtain

$$
\operatorname{Pr}\left(\left\{\sum_{i=1}^{m} \max _{j<i}\left|\left\langle\chi_{R B_{2}^{k}} h_{i}, h_{j}-x\right\rangle\right| \geq 2 R(r+|x|) \lambda\right\}\right) \leq e^{-\lambda^{2} /(8 m)}
$$

for every $\lambda \geq c_{0} m \sqrt{(\ln m) / k}$.

Lemma 2.3 Let $k \leq n \leq m$ be positive integers, $y \in 2 B_{2}^{n}$. Let $h_{1}, \ldots, h_{m}$ be independent random vectors uniformly distributed on $S^{n-1}$. Let $P$ be an orthogonal projection of rank $k$. Denote $v_{i}=P h_{i}, i \leq m$, and $x=P y$. Then there are absolute constants $c, c_{1}$ such that for every $1 \leq A \leq c_{1} k / \sqrt{n \ln m}$ we have

$$
\operatorname{Pr}\left(\left\{v_{i} \in A \text { absconv }\left\{v_{j}-x\right\}_{j \neq i}+\frac{\sqrt{k}}{16 \sqrt{n}} P B_{2}^{n}, \text { for all } i\right\}\right) \leq 2 e^{-c m k /\left(n A^{2}\right)}
$$

Proof: Denote $\bar{v}_{i}=v_{i} \chi_{B}, i \leq m$, where $B=2 \sqrt{k / n} P B_{2}^{n}$. Clearly, if $v_{i} \in A$ absconv $\left\{v_{j}-x\right\}_{j \neq i}+\frac{\sqrt{k}}{16 \sqrt{n}} P B_{2}^{n}$ then $\bar{v}_{i} \in A$ absconv $\left\{v_{j}-x\right\}_{j \neq i}+$ $\frac{\sqrt{k}}{16 \sqrt{n}} P B_{2}^{n}$, which means that there exist $\lambda_{j} \in[-1,1]$ with $\sum\left|\lambda_{j}\right| \leq 1$ and $z \in \frac{\sqrt{k}}{16 \sqrt{n}} P B_{2}^{n}$ such that

$$
\bar{v}_{i}=A \sum_{j \neq i} \lambda_{j}\left(v_{j}-x\right)+z .
$$

Taking scalar product with $\bar{v}_{i}$ we obtain

$$
\left\langle\bar{v}_{i}, \bar{v}_{i}\right\rangle \leq A \sum_{j \neq i}\left|\left\langle\bar{v}_{i}, \lambda_{j}\left(v_{j}-x\right)\right\rangle\right|+\left|\left\langle\bar{v}_{i}, z\right\rangle\right| \leq A \max _{j \neq i}\left|\left\langle\bar{v}_{i}, v_{j}-x\right\rangle\right|+\frac{k}{8 n} .
$$

Therefore if $v_{i} \in A$ absconv $\left\{v_{j}-x\right\}_{j \neq i}+\frac{\sqrt{k}}{16 \sqrt{n}} P B_{2}^{n}$ for every $i$ then $\left|\bar{v}_{i}\right|^{2}-\frac{k}{8 n} \leq$ $A \max _{j \neq i}\left|\left\langle\bar{v}_{i}, v_{j}-x\right\rangle\right|$ for every $i$, which implies

$$
\sum_{i=1}^{m}\left|\bar{v}_{i}\right|^{2}-\frac{k m}{8 n} \leq A \sum_{i=1}^{m} \max _{j \neq i}\left|\left\langle\bar{v}_{i}, v_{j}-x\right\rangle\right| .
$$


The probability of the last event is less than or equal to

$$
\begin{aligned}
& \operatorname{Pr}\left(\left\{\sum_{i=1}^{m}\left|\bar{v}_{i}\right|^{2} \leq \frac{k m}{4 n}\right\}\right) \\
& \quad+\operatorname{Pr}\left(\left\{\frac{k m}{8 n A} \leq \sum_{i \leq m} \max _{j<i}\left|\left\langle\bar{v}_{i}, v_{j}-x\right\rangle\right|+\sum_{i \leq m} \max _{j>i}\left|\left\langle\bar{v}_{i}, v_{j}-x\right\rangle\right|\right\}\right) .
\end{aligned}
$$

Clearly $\mathbf{E}\left|v_{i}\right|^{2}=\frac{k}{n}$. Thus, using (2), one can see that $\mathbf{E}\left|\bar{v}_{i}\right|^{2} \geq \frac{k}{2 n}$. Since $\bar{v}_{i} \in 2 \sqrt{k / n} B_{2}^{n}$, we have also that $\left\|\left|\bar{v}_{i}\right|^{2}\right\|_{\infty} \leq 4 k / n$. Applying (4) we obtain

$$
\operatorname{Pr}\left(\left\{\sum_{i=1}^{m}\left|\bar{v}_{i}\right|^{2} \leq \frac{k m}{4 n}\right\}\right) \leq e^{-c m}
$$

for an absolute constant $c$. The second term in (6) is bounded by $e^{-c_{1} m k /\left(A^{2} n\right)}$ because of estimate (5) with $r=1, R=2 \sqrt{k / n}, \lambda=\frac{\sqrt{k} m}{96 \sqrt{n} A}$. That proves the lemma.

Proof of Theorem 2.1: Let us note that it is enough to prove the Theorem for orthogonal projections only. Indeed, let $P$ be any projection. Denote by $Q$ the orthogonal projection with the same kernel. Clearly, $Q$ has the same rank as $P, Q P=Q$ and thus if $P K$ is $A$-symmetric then $Q K$ is $A$-symmetric as well.

Let $m \in \mathbb{N}, m=\left[c_{0} n^{3}\right]$, and $\delta=c / \sqrt{n}$, where $c_{0}$ and $c$ are absolute constants, which will be chosen later.

Given $k \leq n$ let $A_{k}>1$ be parameter, which will be specified later $\left(A_{k} \approx k / \sqrt{n \ln n}\right)$.

Let $\mathcal{N}_{k}$ be a $\delta$-net of projections of rank $k$. Let $\mathcal{M}$ be a $\delta$-net in $B_{2}^{n}$. By Fact 1.2 and the remark that follows, we can assume that $\left|\mathcal{N}_{k}\right| \leq(6 / \delta)^{n^{2}}$ and $|\mathcal{M}| \leq(3 / \delta)^{n}$.

First we show that there exist vectors $h_{1}, h_{2}, \ldots, h_{m}$ in $\mathbb{R}^{n}$ such that for every $k \leq n$, satisfying $A_{k}>1$, for every $Q \in \mathcal{N}_{k}$ and every $b \in \mathcal{M}$ the following condition does not hold at least for one $i \in\{1,2, \ldots, m\}$

$$
-Q h_{i} \in A_{k} \operatorname{conv}\left\{Q\left(h_{j}-x_{b}\right)\right\}_{j \neq i}+2\left(A_{k}+1\right) \delta Q B_{2}^{n},
$$

where $x_{b}=\left(1+1 / A_{k}\right) Q b \in 2 B_{2}^{n}$. 
Indeed, consider independent random vectors $h_{1}, \ldots, h_{m}$ uniformly distributed on $S^{n-1}$. Then by Lemma 2.3 the probability that $(7)$ holds for every $i$ with fixed $Q$ and $b$ is less than

$$
\operatorname{Pr}(\{(7) \text { holds } \forall i\}) \leq 2 e^{-c_{1} m k /\left(n A_{k}^{2}\right)}
$$

for $A_{k} \leq c_{2} k / \sqrt{n \ln m}$, where $c_{1}, c_{2}$ are absolute constants. Take $A_{k}=$ $c_{2} k / \sqrt{n \ln m}$. Then $2\left(A_{k}+1\right) \delta \leq \sqrt{k} /(16 \sqrt{n})$ for an appropriate choice of an absolute constant $c$. Therefore probability that there exist $Q \in \mathcal{N}_{k}, b \in \mathcal{M}$ such that the inclusion (7) holds for every $i$ is less than

$$
\begin{aligned}
\sum_{Q \in \mathcal{N}_{k}} \sum_{b \in \mathcal{M}} & \operatorname{Pr}\left(\left\{\forall i: Q h_{i} \in A_{k} \operatorname{absconv}\left\{Q h_{j}-x_{b}\right\}_{j \neq i}+\frac{\sqrt{k}}{16 \sqrt{n}} Q B_{2}^{n}\right\}\right) \\
& \leq 2\left(\frac{6}{\delta}\right)^{n^{2}}\left(\frac{3}{\delta}\right)^{n} e^{-c_{1} m k /\left(n A_{k}^{2}\right)} \\
& \leq 2 \exp \left(2 n^{2} \ln (6 \sqrt{n} / c)-c_{1} m \ln (m) /\left(c_{2}^{2} k\right)\right) .
\end{aligned}
$$

Thus there are absolute constants $c_{0}, c_{3}, c_{4}$ such that for $m=\left[c_{0} n^{3}\right]$ the probability

$$
\begin{aligned}
& \operatorname{Pr}\left(\left\{\exists k \exists Q \in \mathcal{N}_{k}, \exists b \in \mathcal{M} \text { such that (7) holds } \forall i\right\}\right) \\
& \leq \sum_{k} \operatorname{Pr}\left(\left\{\exists Q \in \mathcal{N}_{k}, \exists b \in \mathcal{M} \text { such that (7) holds } \forall i\right\}\right) \\
& \leq 2 n \exp \left(-c_{3} n^{2} \ln n\right) \leq \exp \left(-c_{4} n^{2} \ln n\right) .
\end{aligned}
$$

Hence we can find vectors $h_{1}, h_{2}, \ldots, h_{m}$ with the desired property. Moreover, the random choice gives such vectors with probability larger than $1-\exp \left(-c_{4} n^{2} \ln n\right)$.

Now take such vectors and set $B=\operatorname{conv}\left\{h_{i}\right\}_{i \leq m}$. Fix $k$. Let $P$ be an orthogonal projection of rank $k, A=A_{k}$. Then the body $P B$ is $A$-symmetric with respect to some center $a$ if and only if $-P h_{i}+a \in A\left(\operatorname{conv}\left\{h_{j}\right\}_{j \leq m}-a\right)$ for every $i \leq m$. The last inclusion implies

$$
-P h_{i} \in A \operatorname{conv}\left\{P h_{j}\right\}_{j \neq i}-(A+1) a
$$

for every $i$. By the definition of $\delta$-net there are $Q \in \mathcal{N}_{k}$ and $b \in \mathcal{M}$ such that $\|Q-P\| \leq \delta$ and $|a-Q b| \leq \delta$. Therefore $\max _{i}\left|(Q-P) h_{i}\right| \leq \delta$ and

$$
\begin{aligned}
& \operatorname{dist}\left(-Q h_{i}, A \text { conv }\left\{Q h_{j}\right\}_{j \neq i}-(A+1) Q b\right) \\
& \leq \delta+A \delta+(A+1) \delta=2(A+1) \delta .
\end{aligned}
$$


This means that for some $Q \in \mathcal{N}_{k}$ and $b \in \mathcal{M}$ the inclusion (7) holds for every $i$. That contradicts with the choice of $\left\{h_{i}\right\}$. Thus we obtain that $P B$ is not $A$-symmetric for $A=c_{2} k / \sqrt{n \ln m}$. This proves the theorem.

\section{References}

[A] K. Azuma, Weighted sums of certain dependent random variables, Tôhoku Math. J. 19 (1967), no. 2, 357-367.

[GGM] Y. Gordon, O. Guédon, M. Meyer, An isomorphic Dvoretzky's theorem for convex bodies, Studia Math. 127 (1998), no. 2, 191-200.

[G1] E. D. Gluskin, The diameter of Minkowski compactum roughly equals to n, Funct. Anal. Appl. 15 (1981), 57-58 (English translation).

[G2] E. D. Gluskin, Finite-dimensional analogues of spaces without a basis, (Russian) Dokl. Akad. Nauk SSSR 261 (1981), no. 5, 1046-1050.

[Gr] B. Grünbaum, Measures of symmetry for convex sets, Proc. Sympos. Pure Math. 1963, Vol. VII pp. 233-270 Amer. Math. Soc., Providence, R.I.

[LT] A. E. Litvak, N. Tomczak-Jaegermann, Random aspects of highdimensional convex bodies, GAFA Israeli Seminar, Lecture Notes in Math., V. 1745, 169-190, Springer-Verlag, 2000.

[LMP $]$ A. E. Litvak, V. D. Milman, A. Pajor, The covering numbers and "low $M^{*}$-estimate" for quasi-convex bodies. Proc. Amer. Math. Soc., 127 (1999), 1499-1507.

[MT] P. Mankiewicz, N. Tomczak-Jaegermann, Quotients of finite-dimensional Banach spaces; random phenomena, in "Handbook in Banach Spaces", (eds. W. B. Johnson, J. Lindenstrauss), Elsevier, to appear.

[MP] V. D. Milman, A. Pajor, Entropy and asymptotic geometry of nonsymmetric convex bodies. Advances in Math., 152 (2000), no. 2, 314-335; see also Entropy methods in asymptotic convex geometry. C.R. Acad. Sci. Paris, S.I. Math., 329 (1999), no. 4, 303-308.

[MS] V. D. Milman, G. Schechtman, Asymptotic theory of finite-dimensional normed spaces, Lecture Notes in Math., 1200, Springer, Berlin-New York, 1985. 
[R1] M. Rudelson, Sections of the difference body, Discrete Comput. Geom. 23 (2000), no. 1, 137-146.

[R2] M. Rudelson, Distances between non-symmetric convex bodies and the $M M^{*}$-estimate, Positivity, 4 (2000), no 2, 161-178.

[Sz] S. J. Szarek, Nets of Grassmann manifold and orthogonal group, Proceedings of research workshop on Banach space theory (Iowa City, Iowa, 1981), 169-185, Univ. Iowa, Iowa City, Iowa, 1982.

Department of Mathematics,

Tel Aviv University

Ramat Aviv, Tel Aviv

Israel, 69978

gluskin@math.tau.ac.il

Department of Mathematics,

Technion, Haifa

Israel, 32000

alex@math.technion.ac.il

Department of Mathematical Sciences,

University of Alberta,

Edmonton, Alberta,

Canada T6G 2G1,

ntomczak@math. ualberta.ca 